\title{
A Study on PHM Method Suitable for Motor-Driven Commercial Vehicle
}

\author{
Hong Suk Chang ${ }^{1}$, Jong Chan Park ${ }^{2}$ \\ ${ }^{1,2}$ Hyundai Motor Company, Hwaseong, 18280, Republic of Korea \\ paperclock@hyundai.com
}

\begin{abstract}
Commercial vehicles need to use PHM because the drivers cannot earn money during that period when a breakdown occurs. However, PHM technology has been actively applied in aviation and military, but it is also true that it has never been properly applied due to various limitations in automobile industry. In this study, PHM methodology suitable for commercial vehicle is constructed.

In the first step, the type, number, and position of the sensors are selected by applying the optimization technique so that the failure mode can be detected with considering cost reduction. Second, in case of a single vehicle such as aviation or military, PHM could be applied by using only data acquisition. However, in the case of commercial vehicles with several derivative cars, a hybrid model that considers the analytical model and data acquisition was applied to construct a diagnostic and prognostic model. Lastly, until now data driven approach was a way to check the status with the data obtained from the sensor and to report the remaining useful life when it continued. In advanced, in the vehicle, a dynamic model should be used to take into account external environment. And the SOFT SENSOR methodology that can catch the state of a specific weak point by using the detailed analysis model of $\mathrm{NVH}$, thermal, and strength has been constructed.
\end{abstract}

\section{INTRODUCTION}

Before describing the PHM (Prognostics and Health Management) technology, it started from the point of view of logistics management to efficiently manage the parts so that the equipment used in aviation or military service should be repaired by providing parts in a timely manner. PHM is a new technology that grasps the condition and performs proper maintenance before the equipment breaks Hong Suk CHANG This is an open-access article distributed under the terms of the Creative Commons Attribution 3.0 United States License, which permits unrestricted use, distribution, and reproduction in any medium, provided the original author and source are credited. down. PHM technology is a way to increase equipment utilization and reduce maintenance costs, rather than repairing equipment immediately by parts which is prepared for failure.

PHM technologies are widely spreading to structures as a SHM $^{1)}$ (Structural Health Monitoring), and moving vehicles as a $\operatorname{IVHM}^{2)}$ (Integrated Vehicle Health Management). In particular, the need for smart factories has been recognized due to the 4th Industrial Revolution. The utilization of PHM is accelerating as the operation and maintenance management is improved by installing the IoT(Internet of Things) on real operating equipment. ${ }^{3)}$

The number of applications of PHM in the facility field is increasing. It could reduce the failure rate by detecting manufacturing process errors and determining whether the facilities used in the factory are defective. The aerospace industry is the only field where PHM is actively applied to moving vehicles rather than stationary objects such as equipment.

PHM includes diagnostic and prognostic technologies. In the case of diagnostic technology, we can determine if there is any failure by comparing the measured signal with the fault feature classified as a reference signal or experience. In the case of prognostic technology, we can derive the remaining useful life to failure once an acceptable performance degradation range is established by assuming that the measurement can be used to check the performance degradation. As mentioned earlier, the topics and approaches about PHM applications are steadily researched, but PHM technology for vehicles has not been achieved so far.

Commercial vehicles are very diverse. There are dozens of buses, including school buses, express buses and city buses. And there are dozens of trucks carrying soil, sand or rock. However, most companies are characterized by the fact that their usage is not changed if they are owned by the transportation, logistics or the individual. That is, the accuracy of the residual life prediction is increased due to the use of the same pattern. And, as you know from the 
commercial point of view, if the vehicle is broken, it will take a wasteful time and extra cost for towing, parts supply and repair, and profit will be lost during that period. Therefore, as PHM technology for commercial vehicles is developed, regular diagnostic will inform the drivers the vehicle condition can shorten downtime with scheduled maintenance. Predictive maintenance will provide significant benefits in the view of total cost of ownership (TCO).

In this study, we will introduce the individual research elements required to establish the PHM method for commercial vehicles, and explain some results related to PHM.

\section{MAIN}

\subsection{Research fields required for commercial vehicle PHM}

There are three research fields that are needed to develop PHM technology suitable for commercial vehicles. The first is to prioritize which fault mode is the most problematic in a newly developed product and to find a countermeasure for how to deal with each failure mode. Second, unlike the conventional PHM method used in aviation, logistics, and facilities, a new approach has to be developed for applying it to a number of derivative commercial vehicles. This is because it is impossible to create a model for the diagnostic and prognostic of all derivatives. Finally, the condition of the system has to be detectable by using the signal obtained from the vehicle in driving. To do this, we need to understand the behavior of the vehicle and isolate the vehicle motion and signals to make a clear diagnostic.

In this study, only the progress of the first and second research fields will be explained.

\subsection{FTA(Fault tree analysis) and sensing strategy}

\subsubsection{System Modeling and FMEA(Failure Mode Effect Analysis)}

A vehicle is composed of several systems, which are composed of several tens of components. It is necessary to establish a procedure for selecting items suitable for PHM in a full vehicle having several layers and numerous parts. When developing a new system, each company are proceeding with FMEA $^{4)}$ (Failure Mode Effect Analysis) internally to derive several failure modes and countermeasures corresponding to each failure mode.

In the conventional method, it is common for the test, analysis, and design persons to gather a few days to analyze the relevant system, to instantly derive the concerned failure modes for each system, to plan how to deal with them individually. After all, it was common to leave only documents without checking for follow-up work.

In this study, we were able to analyze the interactions between components and construct models using the concept of system modeling in Figure 1. This model can be effectively used because it utilizes interactions when constructing analytical models for vehicle performance check during vehicle development.

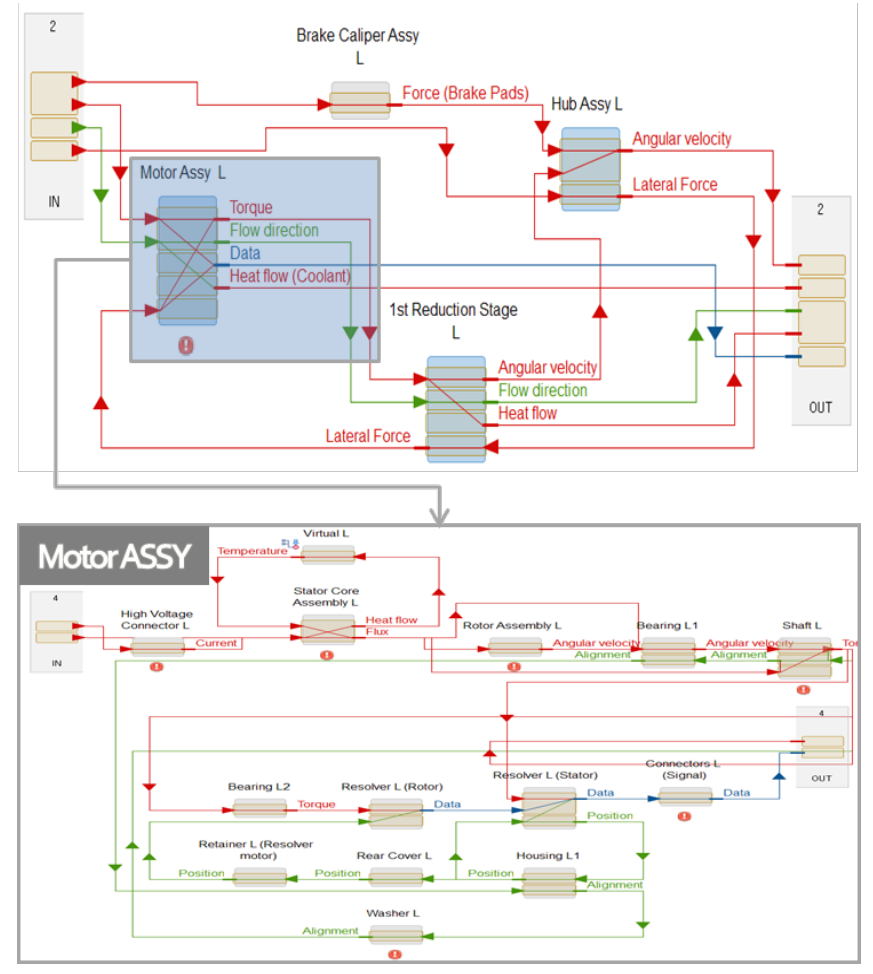

Figure.1 System modeling for FMEA

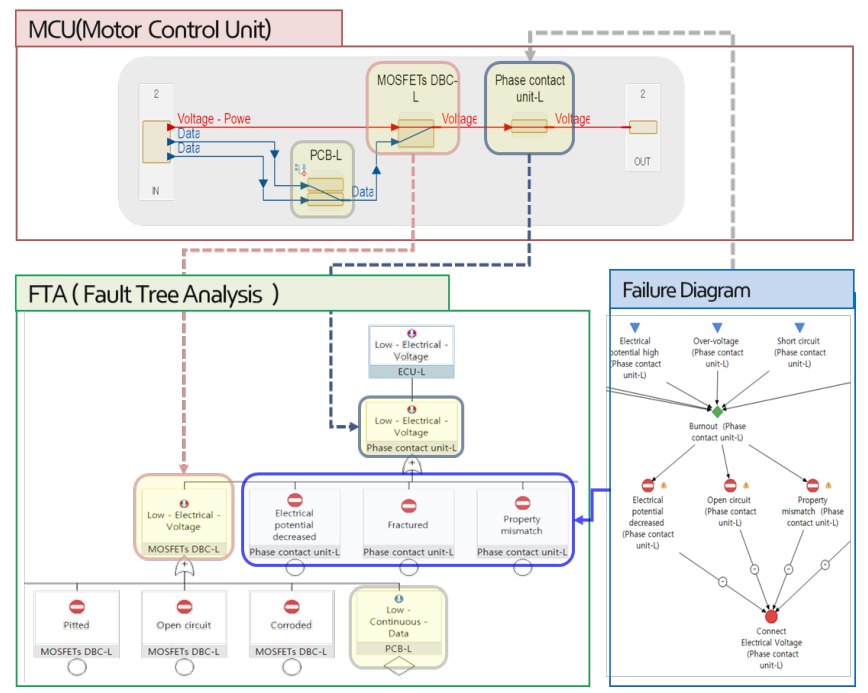

Figure.2 FTA considering Failure Diagram 
In addition, in the derivation process of the failure mode, the failure mode can be derived without brainstorming using the library. And by constructing the target model with the system model, we can understand the influence of the failure mode of each part on other parts. ${ }^{5)}$

In Figure 2, in the Failure Diagram section, we can easily define the available failure modes of each part potentially by referencing to the library. An FTA analysis can be used to comprehensively understand the connection relationship of parts and the failure mode of each part, which can affect the final performance. Although there was a limit to understanding the connection as it progressed by hand, even complex systems could be automatically analyzed using this procedure.

\subsubsection{RPN analysis and sensor set optimization}

Once the failure modes for the target system have been derived, it is necessary to analyze how the failure modes affect the function of the system so that the priority of each failure mode should be determined.

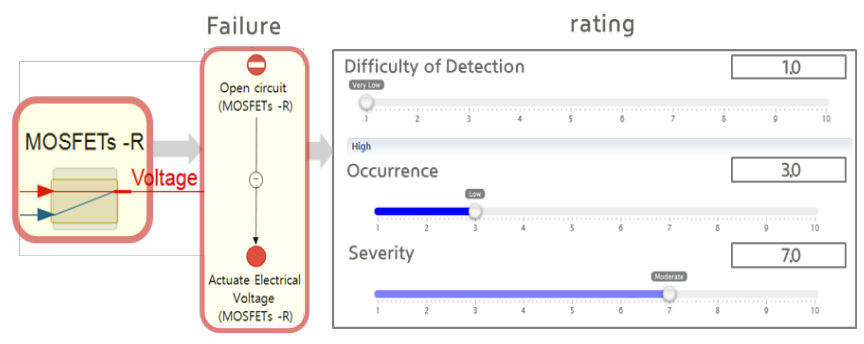

Figure.3 RPN analysis for each Failure Mode

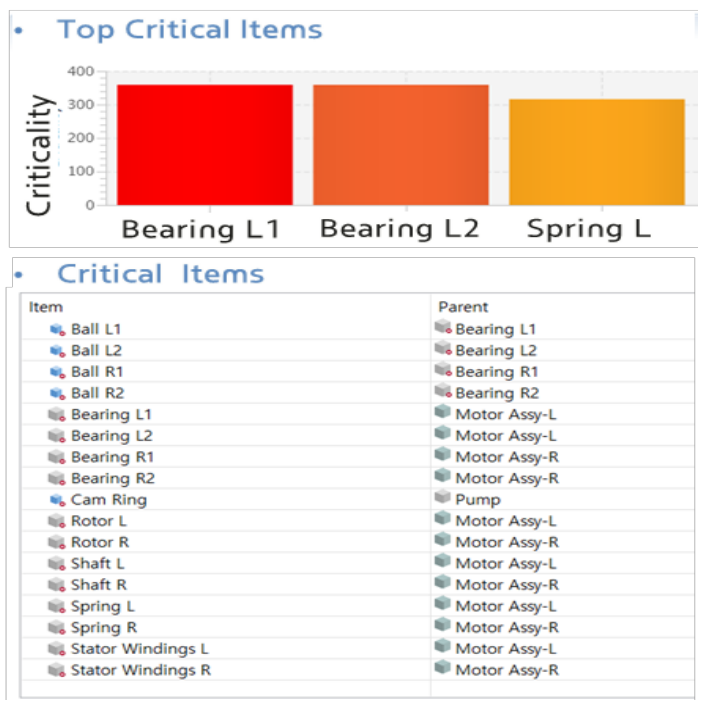

Figure.4 Top Critical Items

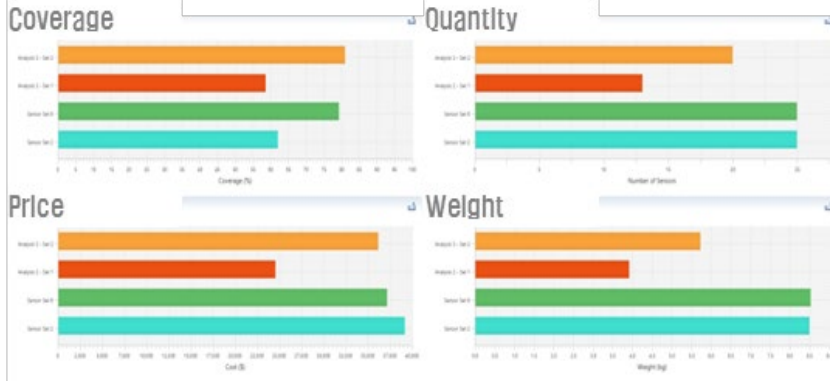

Figure.5 Sensor optimization

People with a high level of understanding of design, testing, and systems should participate in the process of classifying each failure mode and eliminating less important ones. Then, the degree of detection, the degree of occurrence, and the severity of each failure mode are scored based on the SAE J1739 standard.

Major parts and major failure modes are systematically derived through the above process, and next, it is necessary to find a countermeasure for major failure modes. This is called RCM (Reliability Centered Maintenance). 'Do you know the life cycle of the failure mode?' 'Do you know the inspection cycle?' 'Do you know the pre-symptom of the failure?' 'Is it difficult to detect?' It is possible to select appropriate maintenance plan such as scheduled maintenance / conventional maintenance / preventive maintenance considering the period of downtime and repair cost. $^{6)}$

For the items requiring preventive maintenance, it needs to select the sensor to check the condition of the parts. It needs to determine an optimized sensor set considering whether the sensor is in the same fault path, whether the sensor can be installed, the types of sensor needed, price, weight, cost, and the number of sensors appropriate. In some cases, it is necessary to encourage vendors to produce new system installing the necessary sensors in it.

\subsection{Generating diagnostic and prognostic models}

After selecting an item through the process described in the previous section, it needs to generate a diagnostic and prognostic model to diagnose the condition and to know the useful remaining life. Diagnostic and prognostic models are generated by data driven or model based. The main approach has been data driven model using signal data from tests. Recently, many diagnostic models have been developed by collecting large amounts of data in normal and abnormal condition and learning the patterns with machine learning algorithms. For a facility or a single product, datadriven models can be easily implemented because the variables of the external environment are limited and large amounts of data can be easily obtained. However, commercial vehicles have many variables in the external environment, and there are many types of derivative cars to 
which the system applies even if they use the same system. Therefore, there are limits to apply data-driven models to commercial vehicles.

The same type motor used in the commercial vehicle was selected and the motor in the normal condition and the abnormal condition was manufactured referring to the main failure mode obtained through the FMEA. These sections would describe the process of generating diagnostics and prognostic models using data obtained from the tests and the simulations.

\subsubsection{Data driven approach}

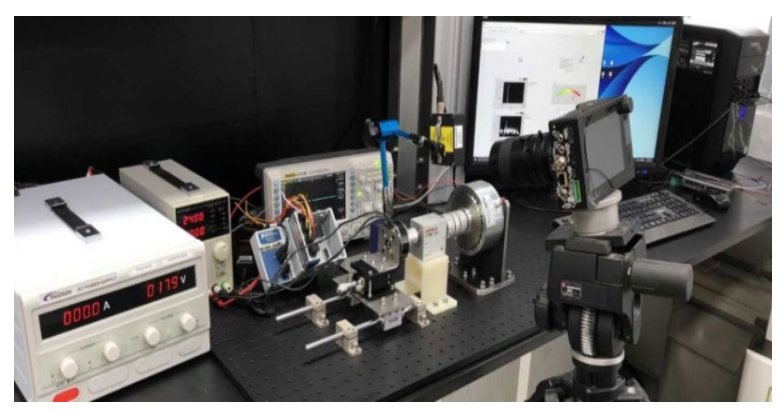

Figure.6 The test devices of Motor System

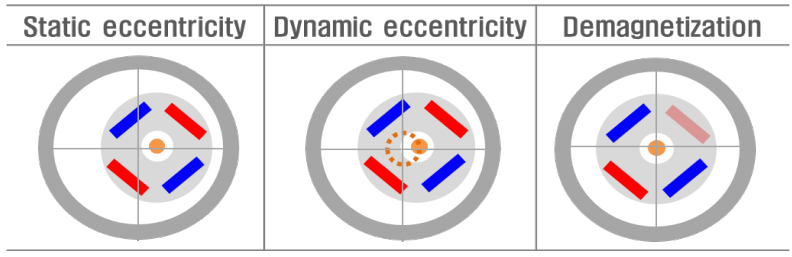

Figure.7 Failure modes for Motor System

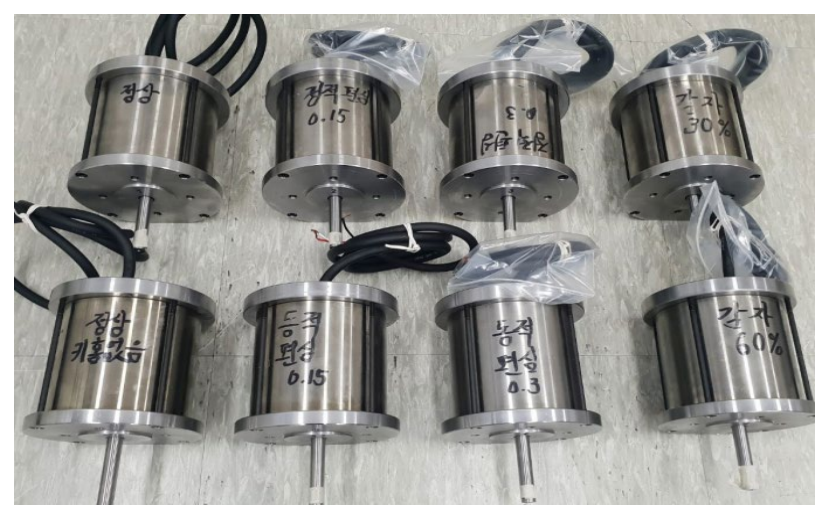

Figure.8 The Motors reflecting Failure Modes

The object of this test is a 3 phase IPMSM (Internal Permanent Magnetic Synchronous Motor) type motor, which is composed of 4poles and 18 slots with two layers of windings. The inverter applies the sinusoidal voltage to drive the motor and the hysteresis brake applies the rotational resistance to control the speed. At this time, a torque sensor for measuring torque, a current probe for measuring current, an accelerometer for acceleration measurement, a microphone for acoustical measurement, and a DAQ device for collecting the measured data were constructed.

The motor supplier has provided the abnormal motors that were customized to achieve static eccentricity, dynamic eccentricity, and demagnetized conditions among several failure modes. Unlike factory-used motors, motors used in vehicles always change RPM from time to time, depending on the conditions. In this test, we analyzed the signals measured under normal and fault conditions while varying the RPM and tried to classify the fault mode using the most discriminating current values. ${ }^{8)}$

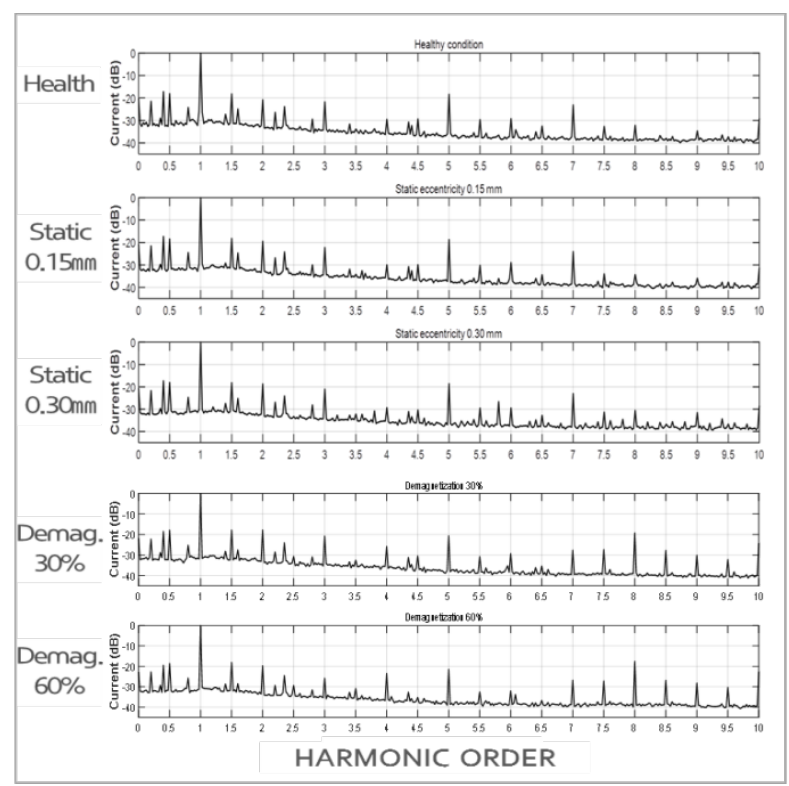

Figure.9 Harmonic Order results of Current Signals

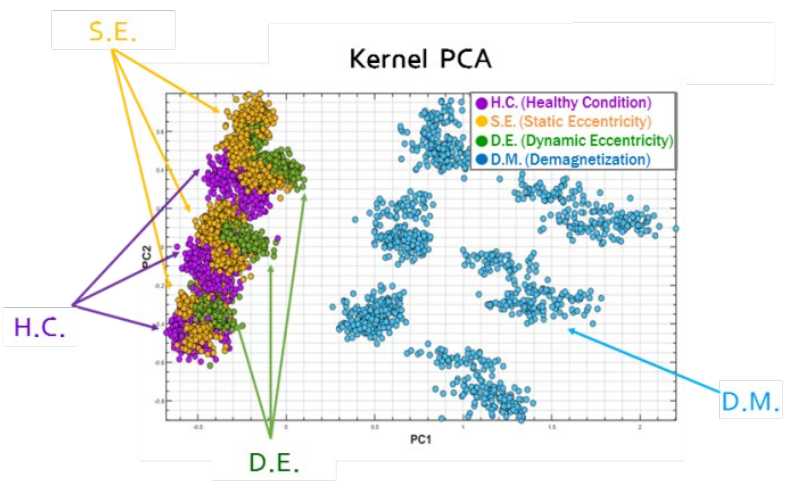

Figure.10 Failure mode classification by KPCA

In figure 9, the first signal procedure to classify fault modes is the order analysis of the current change over time. This is because identifying the change in current in one cycle is the easiest way to classify the fault mode. The following 
procedure is classifying them by Kernel PCA (Principle Component Analysis) method. Kernel is a way to classify by calculating the dot product of the data and arbitrary higher-order functions. The higher order functions include a polynomial kernel, a reproducing kernel, and a sigmoid kernel, and it is necessary to select the best function among them for classification.

As a result of classification by Kernel PCA, the demagnetization condition is clearly classified as shown in the figure 10, but static eccentricity, dynamic eccentricity and health condition are not properly distinguished. It can be recognized that more data is required for data classification and additional data processing is required.

\subsubsection{Model based approach}

Until now, the model-based approach has focused on main functionality, operation, and durability models. In terms of PHM in this area of research, an abnormal model must be built. In this section, an anomaly analysis model was performed to reflect the failure mode based on the previous test for data driven. ${ }^{9)}$

In figure 11 and 12 the structure of the motor is also threephase IPMSM, 4 poles, 18 slots, two layers of windings, and transient analysis is performed to check the current change for each failure mode.

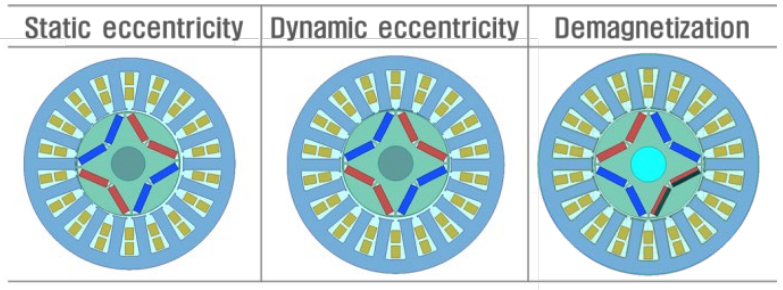

Figure.11 Simulation model for Failure Mode

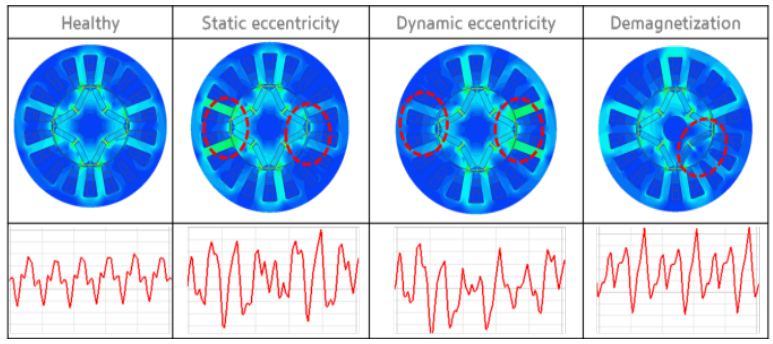

Figure.12 Simulation results for each Failure Mode

As can be seen in Figure 13, the results of the analysis under the failure mode condition reveal that the difference in distance between the stator and the rotor in the case of eccentricity causes a change in the force between the coil and the magnet. As can be seen in Figure 14, in the case of a de-magnetization, it can be seen that the magnet weakens and the force changes even if the distance is constant.
Comparing the signal characteristics of each failure mode based on the order analysis, it can be seen in Figure 15 that the amplitude increases from 1.5th, 2.5th and 3.5th order in both eccentric and demagnetization conditions compared to the health condition. Figure 16 shows that the 2 nd, 4th, 6th order amplitudes are increased under the eccentric condition versus the demagnetization condition. It is also good for capturing a sense of which peak value should be obtained by the signal processing in the test, and it is also possible to predict the pattern change after the model change.

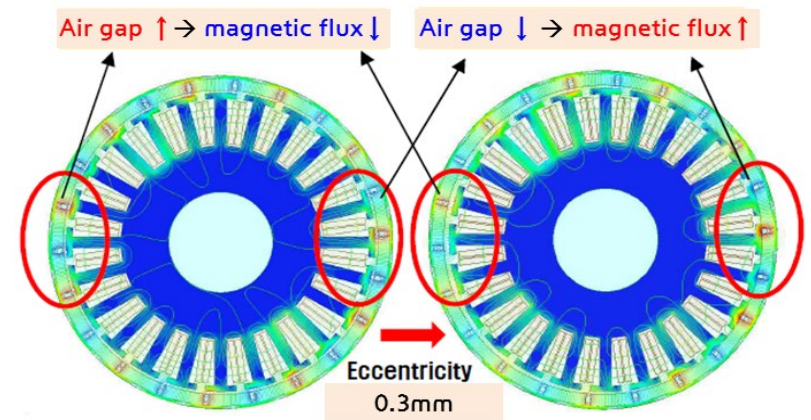

Figure.13 Current Fluctuations by Air-gap

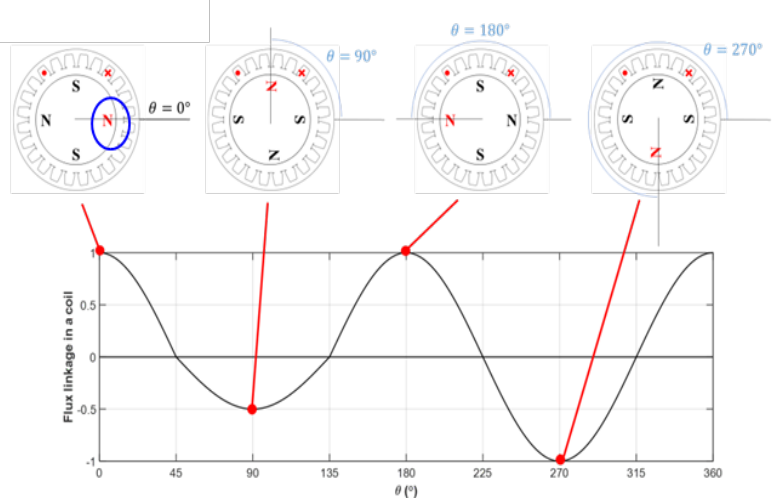

Figure.14 Current Fluctuations by Demagnetization

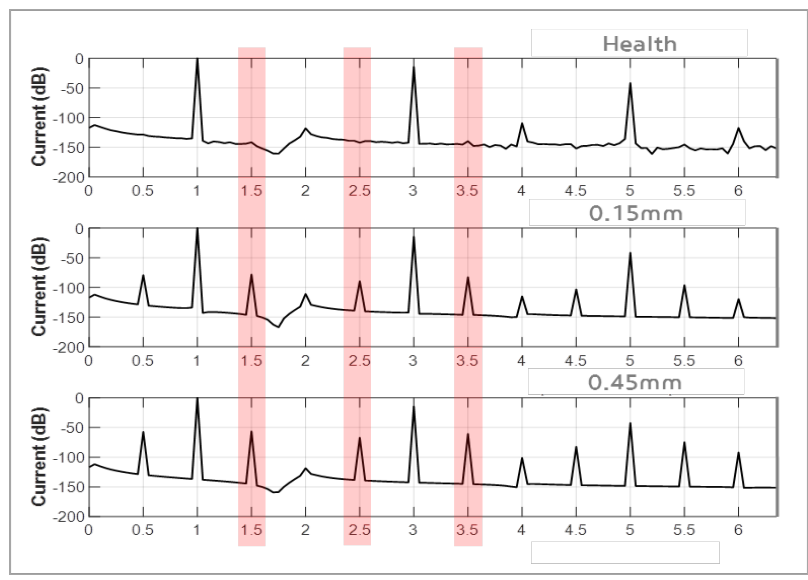

Figure.15 Order Analysis for Eccentricity 


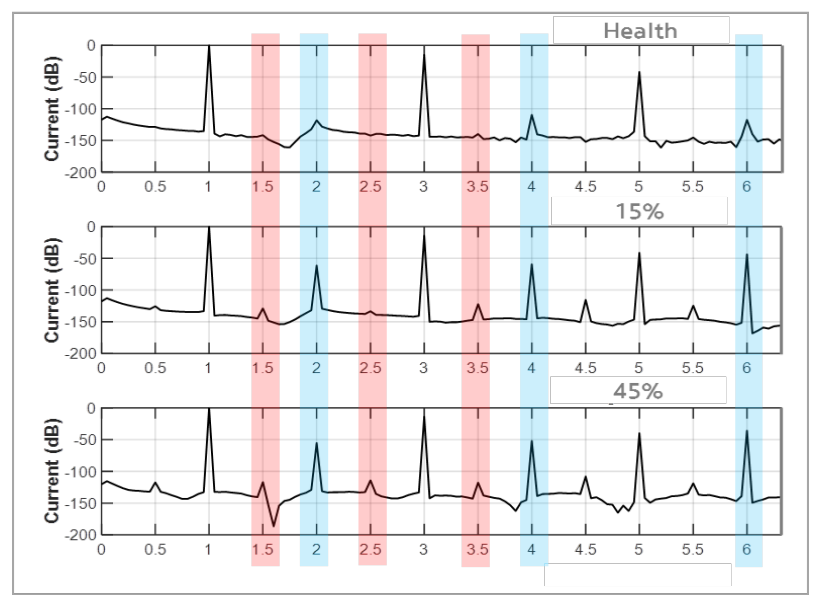

Figure.16 Order Analysis for Demagnetization

\subsubsection{Hybrid Model approach}

In case of data driven, it utilizes a lot of actual signals in the database, so a reliable inference model close to the actual phenomenon can be generated. It is suitable for a single object such as aviation or military because there are a lot of data. The disadvantage is that if there are changes to the model, it needs to collect lots of new signals. The modelbased method is relatively unreliable because it does not utilize actual signals. On the other hand, it has the advantage that it is easy to generate suitable model when the model is changed or even though the commercial vehicle has various environments or derivative vehicles.

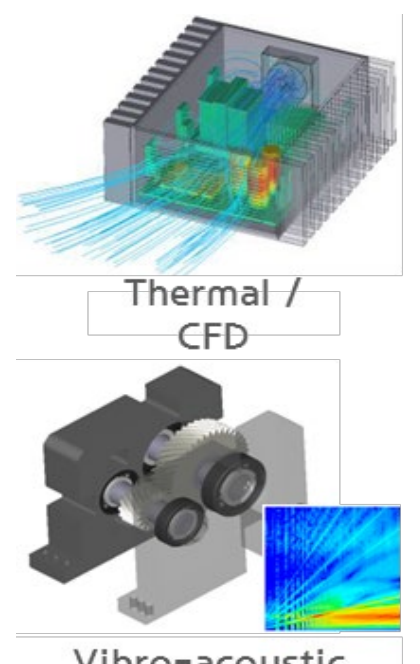

Vibro-acoustic

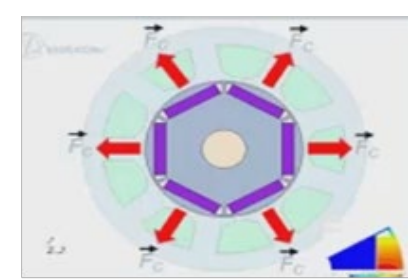

Stress Analysis

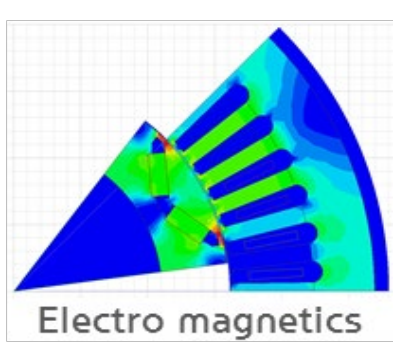

Figure.17 Constructing a Hybrid model using various

Simulation Models (Reference: analysis examples by ANSYS company)

In the vehicle, it's very important to build a hybrid model ${ }^{10)}$ to better utilize the advantages and disadvantages of the above two methods. The methodology is based on a data set that acquires the signals of normal and abnormal condition through the test of the basic motor and establishes the diagnostic and prognostic model of it with reliability. Using the model-based method in conjunction with the above procedure, it's possible to see how the data-based signal changes. This allows us to regenerate the data from the changed model and finally develop the diagnostic and prognostic models of the changed model.

With this procedure, it is possible to access a variety of derivative cars, such as commercial vehicles. In this regard, model-based approaches require various analyzes of target system, so gear strength, NVH analysis, bearing durability analysis, thermal analysis, and electromagnetic field analysis are conducted for normal and abnormal conditions. In addition, the signals would be complex to catch the failure modes, so the signals must be separated considering environmental factors such as road surface, driver characteristics, temperature and vehicle behavior.

Therefore, in order to clarify the diagnostic and prognostic of the system in a moving vehicle, a method of using a dynamic analysis model should be considered to remove the signals from the environmental factors.

\section{CONCLUSION}

In this study, we established a new PHM method suitable for commercial vehicles in order to overcome limitations of conventional PHM methods. The following conclusions were obtained.

1. To select the target system for applying PHM method, system modeling is used to construct the target system and to grasp the function of the individual components and the interaction of the individual components.

2. The failure modes of individual components were identified using the library, and the effect of each failure mode on the target system was analyzed through failure mode effect analysis. As proceeding through the previous process, it could be possible to sort the failure modes that have the greatest influence on the functionality of the target system.

3. The motor which are mainly used for eco-friendly vehicles is target system in this study. The signals obtained from the failure modes (static eccentricity, dynamic eccentricity, and potato phenomenon) were collected and classified based on the data driven approach. And the current signals obtained from the electromagnetic field analysis were also able to be classified by model based approach.

4. By using the simulation model, it was available to understand the principle how to the signals generated to each failure mode, and to study which point should be 
measured to more clearly extract the sensor signal. This process has improved the reliability of diagnostic and prognostic models.

5. A complete analysis of the target system required a variety of simulation models that could be analyzed under abnormal and normal conditions. Therefore, simulation models and analysis for heat transfer, electromagnetic field, strength, NVH and bearing durability of electrical products have been established.

6. Based on this study, is can be available to develop diagnostic and prognostic models suitable for commercial vehicles and also it is possible to apply these results to actual field vehicles.

\section{REFERENCES}

1. Yuan, F.Q. (2016). Structural Health Monitoring (SHM) in Aerospace Structures. Elsevier Ltd.

2. Jennions, I., Niculita, O., Esperon-Miguez, M. (2016). Integrating IVHM and Asset Design, IJPHM.

3. Lee, J., Bagheri, B., Kao, H.A. (2014) Recent Advances and Trends of Cyber-Physical Systems and Big Data Analytics in Industrial Informatics, INDIN.

4. Dyadem Engineering Corporation, (2003) Guideline for Failure Mode and Effects Analysis for Automotive, Aerospace, and General Manufacturing Industries, CRC Press, US.

5. Rudov-Clark, S.D., Stecki, J. (2009) The language of FMEA: on the effective use and reuse of FMEA data, AIAC-13.

6. Igba, J., Alemzadeh, K., Anyanwu-Ebo, I., Gibbons, P., Friis, J.,(2013) A Systems Approach Towards Reliability-Centred Maintenance (RCM) of Wind Turbines, Procedia Computer Science, 814-823.

7. Rudov-Clark, S.D., Ryan, A. J., Stecki, C. M., Stecki, J. S.,(2009) Automated design and optimization of sensor sets for Condition-Based Monitoring, AIAC-13.

8. Ebrahimi, B.M., Faiz, J. (2010) Diagnosis and performance analysis of three phase permanent magnet synchronous motors with static, dynamic and mixed eccentricity, IET Electric Power Applications, 53-66

9. Zhang, A., Bai, Y., Yang, B., Li, H. (2018) Analysis of Nonlinear Vibration in Permanent Magnet Synchronous Motors under Unbalanced Magnetic Pull, applied sciences.

10. Elattar, H.M., Elminir, H.K., Riad, A.M. (2016) Prognostics: a literature review Complex Intell. Syst.

\section{BIOGRAPHIES}

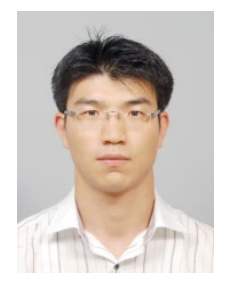

H.S. Chang. is received his B.S degree and M.S degree in Mechanical Engineering from KAIST(Korea Advanced Institute of Science \& Technology) in 2000 and 2002. From 2002 until now, he is a Senior Research Engineer in Hyundai Motor Company. His research interests are in the areas of PHM methodology and Strength and Fatigue of structure.

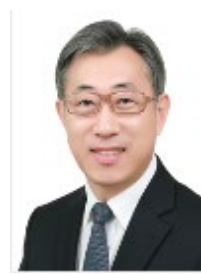

J.C. PARK is received his Ph.D. in Mechanical Engineering from university of Liverpool. He is a Research Fellow in Hyundai Motor Company. 